\title{
Reverse Design o la deconstrucción proyectual del diseño
}

Reverse Design or the projectual deconstruction of design

\author{
> Rodrigo Martin Iglesias \\ Universidad de Buenos Aires, Argentina \\ rodrigo.martin@fadu.uba.ar
}

\begin{abstract}
Reverse engineering is been applied in industry for many years, particularly in the context of the software industry, with the subsequent theoretical development, especially from the 1990s. In this paper we propose to realize their potential application to the field of design, and then characterize the concept of Reverse Design, associated with the idea of projectual deconstruction, concept that has great potential, from didactics to the optimization of materials and energy. Based on a literature review of the state, we will use the basic texts of reverse engineering to establish parallels, differences and contributions to the discipline.
\end{abstract}

Keywords: Reverse Design; Projectual deconstruction; Reverse engineering; Design recovery; Abduction.

\section{Introduction}

"In solving a problem of this sort, the grand thing is to be able to reason backwards. That is a very useful accomplishment, and a very easy one, but people do not practise it much. In the every-day affairs of life it is more useful to reason forwards, and so the other comes to be neglected. There are fifty who can reason synthetically for one who can reason analytically. (...) Most people, if you describe a train of events to them, will tell you what the result would be. They can put those events together in their minds, and argue from them that something will come to pass. There are few people, however, who, if you told them a result, would be able to evolve from their own inner consciousness what the steps were which led up to that result. This power is what I mean when I talk of reasoning backwards, or analytically." (Conan Doyle 2012, pp. 280-281). En este párrafo Sherlock Holmes destaca la dificultad de razonar analíticamente (reasoning backwards), y de algún modo este es el fundamento del presente artículo, la aplicación de instrumentos cognitivos y operativos para razonar hacia atrás sobre los procesos de diseño, que se caracterizan sin duda por el razonamiento de tipo sintético (reason forwards). Para ello usaremos conceptos extraídos de la ingeniería inversa. Cabe aclarar que pretendemos generar aquí un aporte teórico al campo del diseño y que simultáneamente venimos realizando la implementación de estos conceptos en experiencias prácticas que pretendemos exponer en un futuro artículo cuando hayamos analizado y sistematizado sus resultados.

La ingeniería inversa, o reverse engineering, viene aplicándose en la industria desde hace muchos años, con particular profundización en el contexto de la industria del software, con el subsiguiente desarrollo teórico, sobre todo a partir de la década de 1990 . Hoy en día se observa una producción progresivamente mayor de autores chinos y de países pertenecientes al llamado BRICS, sobre todo India y Brasil, lo cual es bastante coherente con las dinámicas geopolíticas de la producción y el consumo. Nos proponemos dar cuenta de las posibilidades de su aplicación al campo del diseño, para luego caracterizar el concepto de Reverse Design, asociado a la idea de deconstrucción proyectual, concepto que presenta un enorme potencial, desde la didáctica hasta la optimización de materiales o energías. Partiendo de una revisión bibliográfica previa del estado de la cuestión, utilizaremos los textos fundamentales de la ingeniería inversa para establecer paralelismos, diferencias y aportes a la disciplina.

Al no existir prácticamente ninguna bibliografía específica sobre Reverse Design (Ling Sha, 2011; Xiuzi Ye, 2008) o deconstrucción proyectual, por ser conceptos híbridos que desarrollamos para entender y dar marco a una propuesta pedagógica y de investigación, nos vemos obligados a desarrollar un marco teórico propio. A tal fin investigamos sobre el concepto de reverse engineering aplicado a la comprensión de diversos objetos de estudio, de hardware o software, así como sobre el concepto de proceso proyectual, entendido como procedimiento que en principio resultaría lógicamente reversible. En este sentido tomamos la abducción como parte fundamental de este proceso y la aplicamos como razonamiento básico en la tarea de deconstrucción proyectual. 


\section{Reverse engineering \& Design}

Luego de una revisión bibliográfica exhaustiva en la que, además de los motores de búsqueda tradicionales como Google, hemos utilizado bases específicas como Cumincad o ACM digital library, llegamos a la conclusión de que actualmente existe cierta confusión conceptual y terminológica entre reverse engineering y métodos específicos como 3D scanning, 3D digitizing o photogrammetry (Wohlers, 1995; Shih, 2002; Maher, 2002). Por otro lado, detectamos cuatro tipos fundamentales de trabajos alrededor de la ingeniería inversa por fuera de aquellos que tratan sobre el software:

a. Sobre las posibilidades y métodos de replicación de productos industriales. En algunos casos también sobre la introducción de innovaciones (Otto, 1996; Montanha, 2007).

b. Sobre el análisis de objetos o sistemas con el propósito de introducir criterios de eficiencia o eficacia en su producción o performance, lo cual incluye algunos casos de aplicación sobre la arquitectura (Shih, 2002; Mahdavi, 1996).

c. Sobre las particularidades técnicas de los diferentes métodos involucrados en la recuperación de información o la replicación, los cual se refiere fundamentalmente a cuestiones alrededor de la precisión y performance de los sistemas CAD/CAM (Ryder, 2002; Schodek, 2005; Várady, 1996).

d. Sobre las posibilidades de aplicación en la recuperación de información de objetos físicos, particularmente aquellos que se consideran de relevancia histórica o patrimonial, lo cual incluye ejemplos arquitectónicos.

El grueso de los trabajos sobre reverse engineering se encuentra situado actualmente en el campo del software y la programación (Cipresso, 2009), incluso aquellos que se refieren a la relación del concepto con el diseńo o la arquitectura (Eilam, 2005; Li Yuzhong, 2011; Messler, 2013). Vale la pena aclarar que existe un fructífero comercio lingüístico entre el campo de la informática y el campo del diseńo, intercambio no exento de transposiciones semánticas basadas la mayoría de las veces en analogías o metáforas, así diseño o arquitectura pueden tener significados diferentes cuando nos movemos dentro de las diferentes áreas del conocimiento (Ingle, 1994). En este sentido, nuestro trabajo puede ser visto como un intento de recuperación de algunos conceptos, o más acertadamente como la construcción de una doble vía de intercambio conceptual, un feedback epistemológico. Desde esta perspectiva tomaremos la influyente taxonomía que Elliot J. Chikofsky y James H. Cross (1990) realizaron sobre el concepto de reverse engineering, en este artículo definen seis conceptos fundamentales relacionados entre sí: forward engineering (ingeniería directa), redocumentation (redocumentación), restructuring (reestructuración), reengineering (reingeniería), reverse engineering (ingeniería inversa) y design recovery (recuperación de diseño). Que iremos definiendo y aplicando en cada caso al campo del diseño y la arquitectura. En la aplicación que actualmente se hace de estos conceptos a los sistemas de software, nos encontramos con que muchos de estos enfoques se aplican a la obtención de un conocimiento básico de un sistema y su estructura. Sin embargo, mientras que el objetivo de hardware tradicionalmente es duplicar el sistema, el objetivo de software es más a menudo obtener una suficiente comprensión a nivel de diseńo para ayudar al mantenimiento, fortalecer las mejoras, o como soporte de sustitución. La ingeniería inversa es básicamente el proceso de analizar un sistema para identificar los componentes del sistema y sus interrelaciones y crear representaciones del sistema en otra forma o en un nivel más alto de abstracción. Mientras que la ingeniería inversa a menudo implica un sistema funcional existente como sujeto de análisis, esto no es un requisito. La ingeniería inversa en sí misma no implica cambiar el sistema o la creación de un nuevo sistema basado en el sistema objeto de ingeniería inversa. Es un proceso de examen, no un proceso de cambio o replicación (Chikofsky y Cross, 1990).

\section{Forward engineering}

El concepto de forward engineering se refiere al proceso de trasladarse desde diseños (o incluso requerimientos) de un alto nivel de abstracción y gran independencia de su implementación, hacia la implementación física de un sistema. No acordamos en el hecho de que el diseño sea la segunda etapa (caracterizada como especificación de la solución) del llamado ciclo de vida (Chikofsky y Cross, 1990), precedido por la especificación del problema (requerimientos) y seguido por la construcción y puesta en marcha del sistema (implementación). Pensamos que esta visión deriva de una visión del diseño como problem solving que resulta anacrónica y reduccionista, visión que no casualmente se encuentra influenciada por la cibernética y las ciencias de la computación (Simon, 2006). Los niveles lógicos y de abstracción involucrados en el proceso solo son constatables a través de representaciones y podemos considerar que todas ellas forman parte del diseño, incluso la llamada implementación final. Es evidente sin embargo que la disminución de la abstracción como tendencia general, así como el mayor compromiso de las representaciones con la implementación caracterizan el curso habitual del proceso de diseño, lo que podríamos llamar forward design.

\section{Reverse engineering}

La ingeniería inversa aparece definida como el proceso de analizar un sistema para: identificar los componentes y sus interrelaciones, así como para crear representaciones bajo otra forma o a un nivel mayor de abstracción. Además estas representaciones tienden a ser menos dependientes de la implementación. Por otro lado, se puede realizar reverse engineering sobre cualquier nivel de abstracción o en cualquier etapa del proceso proyectual. Abarcando todas las etapas, la ingeniería inversa cubre una amplia gama, comenzando de la aplicación existente, puede volver a capturar o recrear el diseńo, hasta descifrar los requisitos de hecho implementados por el sistema (Chikofsky y Cross, 1990). Entre los objetivos habituales de la ingeniería inversa se encuentran: Hacer frente a la complejidad (debemos desarrollar métodos para lidiar mejor con la complejidad de los sistemas y diseńos). Generar puntos de vista alternativos (facilita la generación o regeneración de representaciones gráficas para facilitar el proceso de revisión y verificación). Recuperar información perdida (las modificaciones con frecuencia no se reflejan en la documentación, y si bien no es un sustituto para la preservación de la historia del diseńo, nos permite tener una idea de los sistemas cuando no entendemos lo que hacen o cómo interactúan como un 
sistema). Detectar los efectos secundarios. Sintetizar abstracciones de nivel superior. Y facilitar la reutilización. Se puede considerar que la ingeniería inversa presenta al menos dos subareas destacables: redocumentation y design recovery.

\section{Redocumentation}

La redocumentación es la creación o revisión de una representación semánticamente equivalente con el mismo nivel relativo de abstracción, que son consideradas como visiones alternativas. Es la forma más antigua y simple de los procesos de ingeniería inversa y es considerada por algunos como una versión débil de reestructuración. Se trata de un intento por recuperar documentación sobre el sistema estudiado que existió o pudo haber existido (Chikofsky y Cross, 1990). Podemos afirmar que esta forma de revertir el proceso es la que más se utiliza en el campo del diseño y la arquitectura (incluso la arqueología) y sus ejemplos son rastreables desde al menos el siglo I (con las representaciones que habrían acompańado la obra de Vitrubio o la famosa Forma Urbis) hasta la actualidad. Hoy en día la redocumentación se ve ayudada por tecnología como el scanning $3 \mathrm{D}$ o la fotogrametría digital que posibilitan la recuperación de información invaluable sobre objetos y edificios de los que no se tiene documentación disponible o esta se encuentra incompleta (Maher, 2002; Dritsas, 2013; Várady, 1996).

\section{Design Recovery}

La recuperación de diseño es un subconjunto de la ingeniería inversa en la que el dominio de los conocimientos del campo, la información externa, y la deducción o el razonamiento difuso se ańaden a las observaciones del sistema objeto para identificar abstracciones significativas de nivel superior más allá de las obtenidas directamente mediante el examen del propio sistema existido. El design recovery se distingue por las fuentes y la envergadura de la información que debe manejar: "Design recovery recreates design abstractions from a combination of code, existing design documentation (if available), personal experience, and general knowledge about problem and application domains (...) Design recovery must reproduce all of the information required for a person to fully understand what a program does, how it does it, why it does it, and so forth. Thus, it deals with a far wider range of information than found in conventional software engineering representations or code" (Ted Biggerstaff citado por Chikofsky y Cross, 1990). La bibliografía revisada sobre design recovery (e.g. Ramos Barbero, 2009; Xiuzi Ye, 2008) nos lleva a pensar que la amplitud de objetivos expresada respecto del software no se encuentra en los otros campos de aplicación, sobre todo en lo concerniente a procesos y estrategias de diseño involucrados. Actualmente nos hallamos desarrollando una serie de experiencias de aplicación práctica de este concepto, dentro del más amplio de Reverse Design, sobre casos de arquitectura, con lo cual pretendemos además especificar mejor sus límites teóricos y prácticos, pero podemos adelantar que viene demostrando una potencialidad sorprendente para la comprensión de los proyectos, su manipulación y transformación.

\section{Restructuring}

La reestructuración es la transformación de una forma de representación a otra de un nivel parecido de abstracción sin cambiar el diseño (funcionalidad y semántica). "However, the term has a broader meaning that recognizes the application of similar transformations and recasting techniques in reshaping data models, design plans, and requirements structures. Data normalization, for example, is a data-to-data restructuring transform to improve a logical data model in the database design process. (...) Many types of restructuring can be performed with a knowledge of structural form but without an understanding of meaning." (Chikofsky y Cross, 1990, p.15). Sin duda se trata de lo que llamaremos traducciones intrasemióticas (dentro de un mismo sistema de signos) e intersemióticas (de un sistema de signos a otro), estrategia constitutiva del forward design y que si bien no cumple en sentido estricto con la condición de no cambiar el diseño (la traducción nunca tiene absoluta fidelidad con el original) es fundamental como parte de la tarea mayor del reverse design, al permitir la aproximación abductiva a las posibilidades de representaciones con mayor nivel de abstracción y a la comprensión de las estrategias proyectuales involucradas.

\section{Reengineering}

La reingeniería es el examen y la modificación de un sistema a reconstituir en una nueva forma y la posterior implementación de la nueva forma. Generalmente incluye alguna forma de ingeniería inversa (para lograr una descripción más abstracta), seguida de algún tipo de reestructuración. Esto puede incluir modificaciones con respecto a nuevos requisitos no cubiertos por el sistema original (Chikofsky y Cross, 1990). La reingeniería es la continuación natural de la ingeniería inversa, fuera de la mera copia o replica de un original, y consideramos que el redesign entendido en este sentido debe substituir las nociones de refuncionalización o reutilización (sobre todo en arquitectura) por tratarse de una noción que lleva implícita necesariamente un proceso de comprensión previa del sistema a ser rediseñado.

\section{Reversing Design}

Pretendemos a partir del cruce dialéctico y la hibridación conceptual de estos campos generar el marco teórico específico para el Diseño Inverso. El proceso de invertir el diseño, retroproyectar el proyecto, que a su vez contiene una serie de subprocesos, se encuentra caracterizado por el uso de referentes disciplinares (casos modelo) o analógicos (el pensamiento basado en analogías es clave dentro de los procesos de inferencias creativas), así como por el uso de la re-representación o traducción entre representaciones (intersemióticas e intrasemióticas). Por otro lado, al tratarse de inferencias, el reverse design funcionara en relación constante con el forward design, básicamente como método de contrastación de hipótesis y feedback. El razonamiento abductivo cumple un rol fundamental en el proceso de deconstrucción proyectual. Este rol se encuentra principalmente localizado en la elaboración de las hipótesis particulares y generales sobre la serie de decisiones proyectuales que permitan a su vez la elaboración de hipótesis procedimentales sobre los métodos o estrategias proyectuales utilizados. De todos modos, cabe aclarar que 
estas inferencias se sostienen siempre en representaciones, que a su vez se sostienen en sistemas de signos, con los cual hay un aspecto semiótico representacional intrínseco e ineludible, que por otro lado constituye la característica fundamental del proceso de diseño. Así como en el proceso proyectual el pensamiento abductivo cumple una función determinante, en el proceso de reverse design (Génova, 2004), si bien se presentan razonamientos deductivos e inductivos, el rol que cumple la abducción es clave para entender el proceso de construcción y establecimiento de hipótesis que sostienen el trazado genealógico. Se busca de tal modo una mejor comprensión y definición del proceso de reverse design para poder de este modo tener mayor control al aplicarlo como instrumento.

El proceso proyectual, entendido como anticipación futura, de carácter propositivo y no explicativo, necesita de la elaboración constante de sus propias reglas y requerimientos, es decir que el proceso proyectual no responde a los requerimientos sino los elabora, estas proposiciones hacen necesario el estudio interpretativo de sus componentes específicos y contextuales, marco en el cual el reverse design se torna una herramienta de análisis crítico, más que descriptivo, más aún si se entiende al objeto resultante del proceso proyectual, el Proyecto, como una consecuencia de un proceso de elaboración interna: intratexto y una externa: contexto, que inciden de manera directa y permanente, y no sólo como un conjunto de factores externos a ser tomados en cuenta (Doberti, 2008). Está lógica de razonamiento coincide con lo que Pierce desarrolla como abducción. Múltiples trabajos dan cuenta de cómo esta noción constituye la base epistemológica y metodológica del proceso proyectual: "dado que la proyectación no es un acto, sino un proceso de gestación, habría una serie sucesiva con interferencias abductivas que irían resolviendo." (Sarquis, 2006, p. 236), donde la lógica abductiva, puede distinguirse en tres tipos: hipercodificada (abducciones con alto grado de previsibilidad), hipocodificada (se selecciona entre una posibilidad conocida de reglas) y la creativa: donde la ley es inventada exnovo; y con este mecanismo fundamenta la posibilidad anticipatoria vertida en el proceso proyectual, el proyecto como anticipación futura.

Como proceso proyectual en sí mismo el reverse design ha permitido trabajar el proyecto de una manera técnica fragmentada, invitando a la operación, con libertad e independencia, de los distintos componentes; estos son tratados de manera individual y recompuestos en una nueva relación (Muñoz Cosme, 2008), que articula este con otras operaciones de investigación de las formas resultantes de los proyectos. Por otro lado, ofrece el marco teórico y posibilita operativamente el estudio de los medios técnicos de representación utilizados por el diseñador y su incidencia en el diseño, en sus diferentes etapas y niveles de abstracción, lo cual en el contexto actual resulta un tema crítico, dado el rol cada vez más protagónico que determinados medios técnicos vienen tomando en los procesos proyectuales (Schodek, 2005; Ryder, 2002; Marshall, 1992). Los ciclos de forward y reverse design, entendidos como procesos de síntesis creativa y análisis interpretativo respectivamente, utilizan las mismas estrategias cognitivas (la mera lectura de Conan Doyle nos lo revela) y en cierto sentido constituyen al diseño en su complejidad multidimensional. Creemos que la definición, caracterización, sistematización y aplicación del reverse design contribuirá de forma sustancial a las disciplinas del diseño, incluida la arquitectura, en aspectos que van desde cuestiones cognitivas asociadas al proceso de diseño (toma de decisiones, representación, traducción, etc.) hasta la mejora o rediseño de proyectos existentes, pasando por el amplio potencial pedagógico de la herramienta conceptual.

\section{Acknowledgments}

Agradecemos a la Universidad de Buenos Aires por proporcionar el financiamiento y a la Prof. Beatriz Galán por brindar el marco institucional para esta investigación.

\section{References}

Chikofsky, E. J., Cross, J., (1990, January) Reverse engineering and design recovery: A taxonomy. IEEE Software.

Cipresso, T. (2009) Software Reverse Engineering Education. SJSU Master's Thesis. ProQuest UML. Retrieved from http:// www.reversingproject.info.

Conan Doyle, A. (2012) The Complete Works of Sherlock Holmes. London: Simon \& Schuster (first published 1887).

Doberti, R. (2008) Espacialidades. Buenos Aires: Infinito.

Dritsas, S., and Kang Shua Y., (2013) Undrawable Architecture Digital Workflows for the Conservation of Heritage Buildings and the Discovery of Digital Tectonic. In Open Systems: Proceedings of the 18th International Conference on Computer-Aided Architectural Design Research in Asia, 833-842.

Eilam, E. (2005). Reversing: Secrets of Reverse Engineering. Wiley Publishing.

Génova, G.; Llorens, J., (2004) Métodos abductivos en ingeniería del software. IV Congreso de la Sociedad de Lógica, Metodología y Filosofía de la Ciencia en España. Universidad de Valladolid. España.

Ingle, K., (1994) Reverse Engineering, McGraw-Hill, New York.

Li Yuzhong and Zhou Li. (2011) Study of Virtual Design Based on Reverse Engineering. In Proceedings of the 2011 Third International Conference on Measuring Technology and Mechatronics Automation - Volume 03 (ICMTMA '11), Vol. 3. IEEE Computer Society, Washington, DC, USA, 410-413.

Ling Sha, (2011) The Innovation Design of Product Based on Reverse Engineering, 2011 International Conference on Computer Science and Information Technology (ICCSIT 2011). IPCSIT vol. 51, IACSIT Press, Singapore.

Mahdavi, A., P. Mathew, V. Hartkopf, and V. Loftness. (1996) Bi-directional Inference in Thermal Design. In Design Computation: Collaboration, Reasoning, Pedagogy: ACADIA Conference Proceedings, 133-143. 
Maher, A.M., and M. Burry. (2002) Hybridized Measurement: Interpreting historical images of Sagrada Família Church in Barcelona using CAD-based digital photogrammetry. In Connecting the Real and the Virtual - design e-ducation: 20th eCAADe Conference Proceedings, 448-455.

Marshall, A. D., R. R. Martin, (1992) Computer Vision, Models, and Inspection, World Scientic Publishing, Singapore.

Messler, R. (2013). Reverse Engineering: Mechanisms, Structures, Systems \& Materials. McGraw Hill.

Muńoz Cosme, A. (2008) El proyecto de arquitectura: concepto, proceso y representación. Barcelona: Reverté.

Montanha, Jr.I.R., Ogliari, A., Back, N. (2007) Guidelines for Reverse Engineering Process Modeling of Technical Systems. In Complex Systems Concurrent Engineering. Springer. London. pp. 23-30.

Otto, K.N., Wood, K.L., (1996) A reverse engineering and redesign methodology for product evolution. Proceedings of the 1996 ASME Design Engineering Technical Conferences and Design Theory and Methodology Conference. Irvine, USA.

Pierce, Ch. (1970) Deducción Inducción e Hipótesis. Original de 1878. en: http:/www.unav.es/gep/DeducInducHipotesis.html

Ramos Barbero, B., (2009) The recovery of design intent in reverse engineering problems. Comput. Ind. Eng. 56, 4 (May 2009), 1265-1275.
Ryder, G., Ion, B., Green, G., Harrison, D., Wood, B., (2002) Rapid design and manufacture tools in architecture, Automation in Construction, 11, 279-290.

Sarquis, J. (2003) Itinerarios del proyecto 1 - Ficción epistemológica. Buenos Aires. Nobuko.

Schodek, D. [et Al.], (2005) Approaches and Strategies, Digital Design and Manufacturing, CAD/Cam Applications in Architecture and Design, John Wiley \& Sons: Hoboken.

Shih, N., Wang, P., (2002) The Application of Reverse Engineering for Building Construction Management. Connecting the Real and the Virtual - design e-ducation: 20th eCAADe Conference Proceedings, 338-341.

Simon, H. A., (2006) Las ciencias de lo artificial, Granada: Comares. Traducido del original: The Sciences of the Artificial, Cambridge: The MIT Press, 1996.

Várady, T., R. R. Martin y J. Cox, (1996) Reverse Engineering of Geometric Models - An Introduction.

Wohlers, T., (1995) 3D Digitizers for Engineering. CGW, 18, 3. Retrieved from http://www.wohlersassociates.com/ mar95.html

Xiuzi Ye, Hongzheng Liu, Lei Chen, Zhiyang Chen, Xiang Pan, and Sanyuan Zhang. (2008) Reverse innovative design - an integrated product design methodology. Computer Aided Design, 40, 7 (July 2008), 812-827. 